\title{
Variability of leaf morphology and marker genes of members of the halophila complex collected in Viet Nam
}

\begin{abstract}
Seagrasses are marine angiosperms and adapted to aquatic life several times independently. In tropical as well as in temperate regions they play an important role in the coastal ecosystem. Classification according to morphology remains difficult due to very similar adaptations to the aquatic lifestyle and small simple flowers which are often not even formed. Especially the Halophila genus (Hydrocharitaceae) forms a complex group with an unresolved taxonomy due to overlapping morphological characters and high plasticity leading to many misidentifications. Hence, this led to the hypothesis that phylogenetic analysis on the molecular level may resolve taxonomic classification. The aim of this study is to identify and unambiguously characterize Halophila species collected in Viet Nam using tree- and character-based analysis of rbcL and matK sequences. Results obtained from molecular data and leaf morphology indicate that there are at least three species found in Viet Nam. Topologies based on single locus or combined datasets were similar but not equal. Analysis of rbcL sequences showed lowest species resolution when only Halophila beccarii Aschers was resolved at the Halophila complex. matK shows better resolution with respect to $\mathrm{H}$. beccarii and $\mathrm{H}$. decipiens Ostenfeld. Combined rbcL and matK consensus trees showed the highest species resolution when all species form three distinct clades representative for three difference species including $\mathrm{H}$. beccarii, $\mathrm{H}$. decipiens, and $\mathrm{H}$. ovalis (R. Br.) Hook. f.
\end{abstract}

Keyword: Halophila; MatK; Phylogenetic analysis; RbcL. 